\title{
The recovery from COVID-19: pathophysiology and potential role of pulmonary rehabilitation program
}

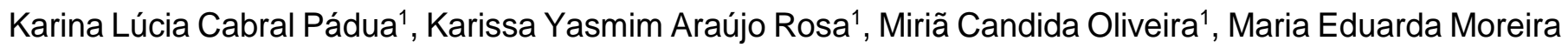
Lino $^{1}$, Adriano Luís Fonseca ${ }^{1}$, Vinícius Zacarias Maldaner ${ }^{1,3}$, Felipe Xavier de Melo ${ }^{1,2}$, Dante Brasil Santos ${ }^{1,2,3}$

${ }^{1}$ Póst Graduate Program in Human Movement and Rehabilitation of Evangelical University of Goiás (UniEVANGÉLICA), Anápolis, Brazil; ${ }^{2}$ Pulmonary Rehabilitation Program, Hospital Universitário de Brasília - Universidade de Brasília (UnB), Brasília, Brazil; ${ }^{3}$ Reference Center of Neuromuscular Diseases, Hospital de Apoio de Brasília, Brasília, Brazil.

\section{ABSTRACT}

Background: The actual stage of SARS-CoV-2 pandemic reveals the necessity to rehabilitate patients, mainly those who were severe and critical ill during acute infection. These post-COVID19 patients might present multiple factors, which culminates in impairment of oxygenation to skeletal muscles, with prejudice in overall functional capacity. These factors can be described as a reduced pulmonary diffusion capacity, a cardiovascular and oxygen transport dysfunction and deconditioning of skeletal muscles. Objective: The objective of the present article is to highlight potential pathophysiological aspects of initial convalescent acute COVID19 and post-acute COVID-19 Syndrome, proposed evaluations and rehabilitation initiatives to these patients following a rehabilitation program and preliminary results from our pulmonary rehabilitation program (PRP). Methods: The proposition of pulmonary rehabilitation program for the aforementioned patients evaluates pulmonary, cardiac, skeletal muscles and overall function. The conduction of this program might address specifically each one of the potential impairment oxygenation factors, with significant impact evaluated functions and, by consequence, in overall functional capacity. Conclusion: The long-term effects of a rehabilitation program on the pulmonary function of these patients are not yet known, since it is a recent disease. However, studies on few survivors of SARS 2003 showed that pulmonary interstitial damage and functional decline caused by SARS are mostly recovered, with a greater extent of recovery within 2 years after rehabilitation

Keywords: COVID-19; pulmonary rehabilitation, pulmonary function; muscular function

\section{BACKGROUND}

The COVID-19 pandemic, caused by infection due to SARS-CoV-2, has been a challenge for health care systems due to its initial severity stages for some patients, but also to related neurological and skeletal muscles manifestations seen after the aforementioned stages. The health care systems, initially pushed to increase an availability on hospitals beds, now are forced to enhance the ability to rehabilitate those patients after stabilization of initial aggressive infection.

Lately, it has been proposed the term postacute COVID-19 Syndrome ${ }^{(1)}$ to describe the persistence of symptoms 4 weeks or more after initial SARS-CoV-2 infection, which can only be explained by its infection. Thus, because of SARS-CoV-2 infection, multiples mechanisms lead to the development of an environment of impaired oxygenation to the tissues, either associated with impaired diffusion, either associated to the transport of oxygen to skeletal muscles, either associated to deconditioning of skeletal muscles, mainly at the severe and critical forms. All of them may play pivotal role for the musculoskeletal functional impairment. Thus, the convalescent acute COVID-19 (recent hospital discharge due to COVID19 hospitalization) and mostly the post-acute COVID-19 patients may present a variety of symptoms, which are addressed as therapy to an integrative rehabilitation program.
The objective of the present article is to highlight potential pathophysiological aspects of initial convalescent acute COVID19 and post-acute COVID19 Syndrome, proposed evaluations and rehabilitation initiatives to these patients following a rehabilitation program and preliminary results from our pulmonary rehabilitation program (PRP).

\section{Physiopathology of post-acute COVID-19 Syndrome, evaluation and pulmonary / physical therapy measures.}

The first pathophysiological mechanism related to post-acute COVID-19 syndrome is the diffusion pulmonary prejudice, which is directly implicated to the occurrence of pneumonia and inflammation on lungs, and promote hypoxemia by perturbation at diffusion capacity and by significant mismatch at ventilation/perfusion ratio. In this sense, the extension of involvement of lungs plays a major role at the emergence of hypoxemia, because of pneumonic and inflammatory process. The patients with post COVID19 syndrome might still present systemic inflammation, dyspnea, reduced exercise capacity, reduced diffusion capacity, restrictive pulmonary syndrome and fibrotic changes on pulmonary images. ${ }^{(1)}$ The second mechanism is due to cardiovascular dysfunction and oxygen transport impairments. With respect to cardiovascular function impairment, it is already 
described an increased cardiometabolic demand, myocardial fibrosis, arrhythmias and autonomic dysfunction, ${ }^{(1)}$ precipitating symptoms as: palpitations, chest pain, dyspnea and reduced capacity of exercise. The presence of angiotensinconverting enzyme type 2 receptor in cardiac tissue leads to direct infection at the heart and also to direct inflammation, at cardiac territory, which is related to the aforementioned reduction in coronary flow, decreases in oxygen supply, and destabilization of coronary plaque and also micro thrombogenesis. ${ }^{(2)}$ Parallel to those derangements there is also a prejudice at transportation of oxygen. In this case, there are descriptions of decrement at hemoglobin counts, and an increase at erythrocyte sedimentation rate..$^{(3,4)}$

The third mechanism is due to deconditioning of skeletal muscles and persistence of state of inflammation. From natural history of evolution of patients with severe acute respiratory syndrome (SARS), for whom the recovery from acute illness may be similar to post-acute COVID19 , we already know that a generalized atrophy with necrosis in muscle fibers, myofibril disarray and $Z$ disc streaming has been reported. ${ }^{(5-8)}$ Furthermore, it seems that there is a decrease of function of both skeletal muscle fibers, type I and II. Clinically, it has been seen that patients with SARS presents a $32 \%$ reduction in handgrip dynamometer; a reduction of $13 \%$ of distance walked in six minutes' Walk Test $(6 \mathrm{MWWT}) ;{ }^{(9)}$ and myalgia and skeletal muscle weakness had also been reported in $25 \%$ to $50 \%$ of symptomatic patients. ${ }^{(10,11)}$ With respect to SARSCoV-2 infection, we already know that during acute phase, the SARS-CoV-2 patients who presented muscular damage presented higher levels of creatinokinase, regardless the severity. ${ }^{(11)}$ It has also been described that the length of stay in hospital is directly related to amount of loss of free fat mass and to the loss at functional independence measure during hospitalization period. ${ }^{(12)}$

Furthermore, it has been described a reduction at distance walked at 6MWT, with respect to predicted values, for $25 \%$ of patients. ${ }^{(13)}$ Parallel to the aforementioned mechanism, there are also peripheral neurologic perturbations due to the systemic inflammation seen in COVID-19 infection, and also due to a "hostile environment" commonly seen in intensive care unit (ICU), i.e., absence of mobility, use of corticosteroid, neuromuscular blocking agents and elevations in blood sugar. Therefore, there seems to be a final environment at muscular level, which may present neuromuscular disorders due to polyneuropathy and/or myopathy, deconditioning and neuromuscular inflammation with necrosis. In this sense, there is a description, related to SARS-CoV-2 infection, which present findings of electroneuromyography in a patient point to a direction of myopathy, ${ }^{(14)}$ i.e., diffuse weakness. Finally, factors as age, muscular functional state before initial infection, drugs during ICU/hospital stay, comorbidities, muscular damage path and nutritional state may contribute for the extension of muscular damage and for the recovery of its function. Finally, these symptoms and pathophysiological mechanisms might be also associated with post-intensive care syndrome, which also present muscular weakness, and then forces to a direction that obligates an integrative rehabilitation approach.

\section{Pulmonary Rehabilitation Program in Post- COVID19}

The minimal proposed evaluation for a rehabilitation program to these patients should be composed by: overall functional evaluation, measured by six minutes-walk test (6MWT); pulmonary function tests (spirometry, diffusion capacity test, arterial blood gas); handgrip dynamometry; skeletal force muscles evaluation by 1 maximal voluntary contraction (1-MVC) for upper and lower limbs; home pulse oximetry; and computed tomography of the chest; echocardiogram and peak exercise ergospirometry test, since clinically appropriated.(1) Those are proposed in order to adapt the protocol of exercise to each patients' condition. Thus, not only functional capacity, but also pulmonary / cardiac functions and overall skeletal muscle function for force generation and maximal aerobic capacity would be evaluated.

From our experience, pulmonary function does not improve with a pulmonary rehabilitation program (PRP), as expected. Thus, a restrictive pattern still remains, even after the conclusion of the program. However, significant improvements were found in 6MWT, 1-MVC in 20 patients followed by our program so far. In this sense, all patients presented an improvement after the PRP at distance walked in 6MWT, and most patients were able to improve more than 100 meters the distance walked from initial evaluation. Additionally, from 4 patients who were initially dependent of supplemental oxygen during exercise, only 1 still presented this dependence, due to a possible pulmonary embolism, identified at medical evaluation before PRP.

PRP should encompasses both aerobic and force exercises, to improve bioenergetics and overall function in type I and type II skeletal muscle fibers, and then global locomotor function of 
patients. It is expected that, for some patients, even without previous history of pulmonary disease, that they might evolve to desaturation at minimal efforts, pointing to the direction that the remaining healthy portion of the lung is able to keep SpO2 under acceptable values (> 92\%) at rest, but when performed a minimal effort, the hypoxemia arises.

For those, the supplemental oxygen therapy and/or even a positive pressure within a respiratory assistance support (RAS), followed by close monitoring should be necessary. From the patients engaged to our program, only 1 still presented a necessity to supplemental oxygen therapy during exercises, even after the conclusion of program. For this patient, we proposed a second round of 8 weeks PRP, with the addiction of RAS during exercises.

Therefore, even in face of diffusion prejudice, we expect a correction and a maintenance at acceptable levels of circulating oxygen during exercise, from the use of these aforementioned resources. Moreover, a proposition of aerobic warm up, followed by resistance training and then a cool-down aerobic routine exercises should be considered. The pathophysiological aspects detailed before and the proposed intervention of PRP are summarized at the Figure 1.

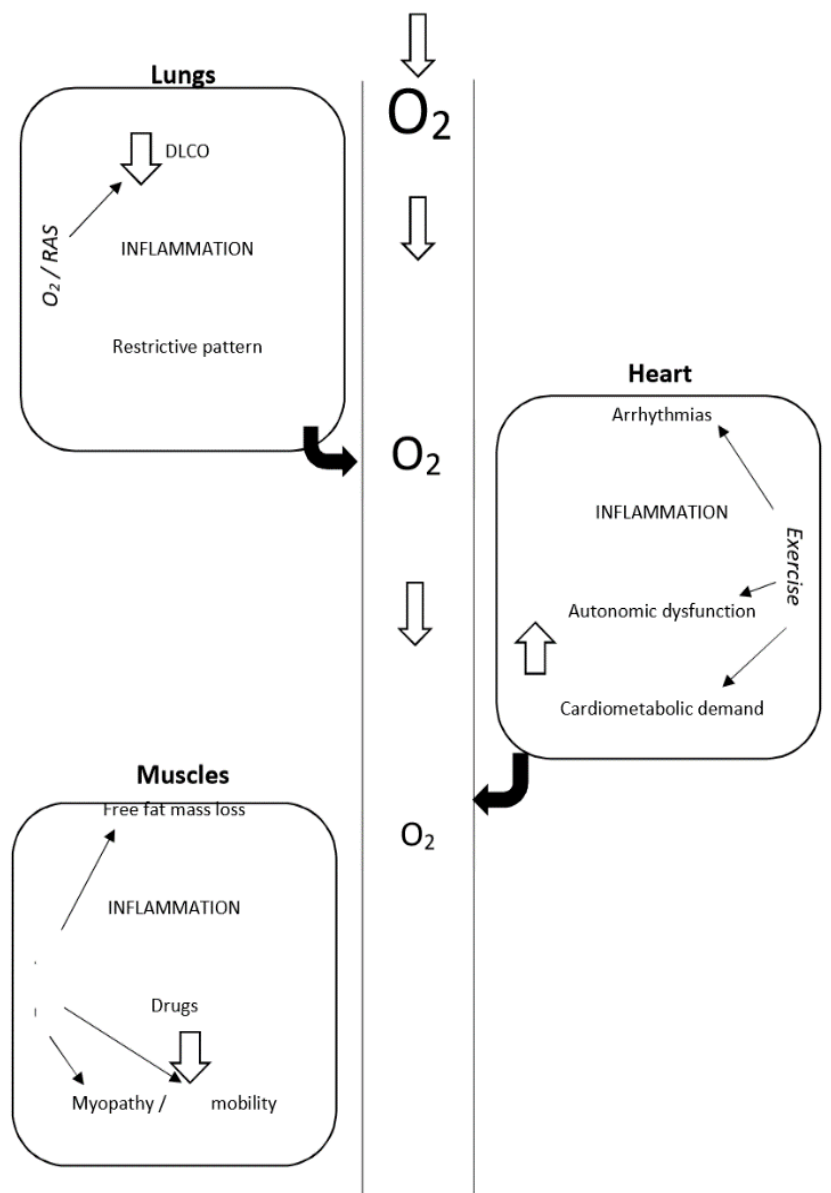

Figure 1. Impairment oxygenation to muscles and potential roles of pulmonary rehabilitation program.

\section{CONCLUSION}

The long-term effects of a rehabilitation program on the pulmonary function of these patients are not yet known, since it is a recent disease. However, studies on few survivors of SARS 2003 showed that pulmonary interstitial damage and functional decline caused by SARS are mostly recovered, with a greater extent of recovery within 2 years after rehabilitation. ${ }^{(15)}$ By analogy, it may be assumed that the same pattern will be seen in some severe and critical cases of COVID-19, which highlights the role of PRP in these patients. Our preliminary data supports the thesis that overall skeletal muscle and functional measurements are improved in an 8-week PRP, reinforcing the importance of the program and the necessity to close follow these patients.

Authors' contributions: KLCP, KYAR, MCO, MEML, ALF, VZM, FXM and DBS contributed for at the revision of the manuscript.

Conflict of interest: There are no potential conflicts of interest with respect to this article.

Financial support: There had no sources of financial support with respect to this article.

\section{REFERENCES}

1. Nalbandian A, Sehgal K, Gupta A, Madhavan MV, McGroder C, Stevens JS, et al. Post-acute COVID-19 syndrome. Nat Med. 2021;27(4):601-15.

2. Huang $\mathrm{C}$, Wang Y, Li X, Ren L, Zhao J, Hu $Y$, et al. Clinical features of patients infected with 2019 novel coronavirus in Wuhan, China. Lancet. 2020;395(10223):497-506.

3. Liu W, Li H. COVID-19:Attacks the 1-Beta Chain of Hemoglobin and Captures the Porphyrin to Inhibit Human Heme Metabolism ChemRxiv. 2020;Preprint.

4. Chen N, Zhou M, Dong X, Qu J, Gong F, Han $Y$, et al. Epidemiological and clinical characteristics of 99 cases of 2019 novel coronavirus pneumonia in Wuhan, China: a descriptive study.

5. Leung TW, Wong KS, Hui AC, To KF, Lai ST, $\mathrm{Ng} \mathrm{WF}$, et al. Myopathic changes associated with severe acute respiratory syndrome: a postmortem case series. Arch Neurol. 2005;62(7):1113-7.

6. Hsiao $\mathrm{CH}$, Chang MF, Hsueh PR, Su IJ. Immunohistochemical study of severe acute respiratory syndrome-associated coronavirus in tissue sections of patients. $J$ Formos Med Assoc. 2005;104(3):150-6. 
7. Disser NP, De Micheli AJ, Schonk MM, Konnaris MA, Piacentini AN, Edon DL, et al. Musculoskeletal Consequences of COVID19. J Bone Joint Surg Am. 2020;102(14):1197-204.

8. Ding $\mathrm{Y}$, Wang $\mathrm{H}$, Shen $\mathrm{H}$, Li Z, Geng J, Han $\mathrm{H}$, et al. The clinical pathology of severe acute respiratory syndrome (SARS): a report from China. J Pathol. 2003;200(3):282-9.

9. Lau HM, Lee EW, Wong CN, Ng GY, Jones AY, Hui DS. The impact of severe acute respiratory syndrome on the physical profile and quality of life. Arch Phys Med Rehabil. 2005;86(6):1134-40.

10. Nasiri MJ, Haddadi S, Tahvildari A, Farsi Y, Arbabi M, Hasanzadeh S, et al. COVID-19 Clinical Characteristics, and Sex-Specific Risk of Mortality: Systematic Review and Meta-Analysis. Front Med (Lausanne). 2020;7:459.

11. Mao L, Jin $H$, Wang $M, H u Y$, Chen $S, H e ~ Q$, et al. Neurologic Manifestations of Hospitalized Patients With Coronavirus Disease 2019 in Wuhan, China. JAMA Neurol. 2020;77(6):683-90.
12. Imamura $M$, Mirisola $A R$, Ribeiro FQ, De Pretto LR, Alfieri FM, Delgado VR, et al. Rehabilitation of patients after COVID-19 recovery: An experience at the Physical and Rehabilitation Medicine Institute and Lucy Montoro Rehabilitation Institute. Clinics (Sao Paulo). 2021;76:e2804.

13. Huang $C$, Huang L, Wang $Y$, Li X, Ren L, Gu $X$, et al. 6-month consequences of COVID19 in patients discharged from hospital: a cohort study. Lancet. 2021;397(10270):22032.

14. Tankisi H, Tankisi A, Harbo T, Markvardsen LK, Andersen H, Pedersen TH. Critical illness myopathy as a consequence of Covid-19 infection. Clin Neurophysiol. 2020;131(8):1931-2.

15. Zhang P, Li J, Liu H, Han N, Ju J, Kou Y, et al. Long-term bone and lung consequences associated with hospital-acquired severe acute respiratory syndrome: a 15-year follow-up from a prospective cohort study. Bone Res. 2020;8:8. 